\title{
Observing the Progressive Decoherence of the "Meter" in a Quantum Measurement
}

\author{
M. Brune, E. Hagley, J. Dreyer, X. Maître, A. Maali, C. Wunderlich, J. M. Raimond, and S. Haroche \\ Laboratoire Kastler Brossel,* Département de Physique de l'Ecole Normale Supérieure, 24 Rue Lhomond, \\ F-75231 Paris Cedex 05, France
}

(Received 10 September 1996)

\begin{abstract}
A mesoscopic superposition of quantum states involving radiation fields with classically distinct phases was created and its progressive decoherence observed. The experiment involved Rydberg atoms interacting one at a time with a few photon coherent field trapped in a high $Q$ microwave cavity. The mesoscopic superposition was the equivalent of an "atom + measuring apparatus" system in which the "meter" was pointing simultaneously towards two different directions - a "Schrödinger cat." The decoherence phenomenon transforming this superposition into a statistical mixture was observed while it unfolded, providing a direct insight into a process at the heart of quantum measurement. [S0031-9007(96)01848-0]
\end{abstract}

PACS numbers: 32.80.-t, 03.65.-w, 42.50.-p

The transition between the microscopic and macroscopic worlds is a fundamental issue in quantum measurement theory [1]. In an ideal model of measurement, the coupling between a macroscopic apparatus ("meter") and a microscopic system ("atom") results in their entanglement and produces a quantum superposition state of the "meter + atom" system. Such a superposition is however never observed. Schrödinger has illustrated vividly this problem, replacing the meter by a "cat" [2] and considering the dramatic superposition of dead and alive animal "states." Although such a striking image can only be a metaphor, quantum superpositions involving "meter states" are often called "Schrödinger cats." Following von Neumann [3], it is postulated that an irreversible reduction process takes the quantum superposition into a statistical mixture in a "preferred" basis, corresponding to the eigenvalues of the observable measured by the meter. From then on, the information contents in the system can be described classically. The nature of this reduction has been much debated, with recent theories stressing the role of quantum decoherence $[4,5]$. According to these approaches, the meter coordinate is always coupled to a large reservoir of microscopic variables inducing a fast dissipation of macroscopic coherences.

The simplest model of a quantum measurement involves a two-level atom $(e, g)$ coupled to a quantum oscillator (meter or cat). An oscillator in a coherent state [6] is indeed defined by a $c$ number $\alpha$, represented by a vector in phase space $(|\alpha|=\sqrt{n}$ where $n$ is the mean number of oscillator quanta). Quantum fluctuations make the tip of this vector uncertain, with a circular gaussian distribution of radius unity [Fig. 1(a)]. Consider the ideal measurement where the "atom-meter" interaction entangles the phase of the oscillator $( \pm \phi)$ to the state of the atom, leading to the combined state

$$
|\Psi\rangle=\frac{1}{\sqrt{2}}\left(\left|e, \alpha e^{i \phi}\right\rangle+\left|g, \alpha e^{-i \phi}\right\rangle\right)
$$

When the "distance" $D=2 \sqrt{n} \sin \phi$ between the meter states is larger than 1, a Schrödinger cat is obtained [Fig. 1(b)].

Decoherence is modeled by coupling the oscillator to a reservoir, which damps its energy in a characteristic time $T_{r}$. When $D \gg 1$, decoherence is found to occur within a time scale $2 T_{r} / D^{2}[7,8]$. This result illustrates the basic feature of the quantum to classical transition [4]. Mesoscopic superpositions made of a few quanta are expected to decohere in a finite time interval shorter than $T_{r}$, while macroscopic ones $(n \gg 1)$ decohere instantaneously and cannot be observed in practice.

Recently, a Schrödinger cat of a material oscillator was generated by preparing a single trapped ion in a superposition of two spatially separated wave packets entangled with internal states of the ion [9]. Quantum decoherence was however not studied. Various schemes have been proposed to prepare Schrödinger cats of a field oscillator [10]. Some of these proposals involve a dispersive coupling between a single atom and a field in free space [11] or in a cavity [8]. Implementing this last scheme, we report here the generation of a Schrödinger cat like state of radiation in a cavity and the first dynamical observation of quantum decoherence in a measurement process.

The mesoscopic state is generated by sending a rubidium atom, prepared in a superposition of two circular Rydberg states $e$ and $g$ [12], across a high $Q$ microwave

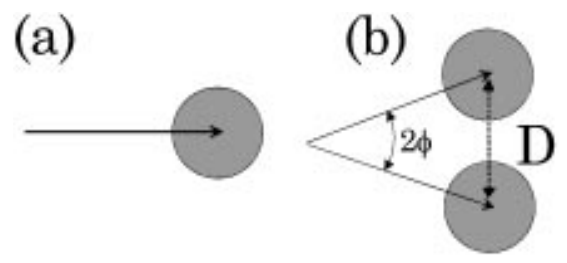

FIG. 1. (a) Pictorial representation in phase space of a coherent state of a quantum oscillator. (b) The two components separated by a distance $D$ of a Schrödinger cat corresponding to Eq. (1). 
cavity $C$ storing a small coherent field $|\alpha\rangle$. The coupling between the atom and the cavity is measured by the "Rabi frequency" $\Omega$ [13]. The $e \rightarrow g$ atomic transition and the cavity frequencies are slightly off resonance (detuning $\delta$ ), so that the atom and the field cannot exchange energy but only undergo $1 / \delta$ dispersive frequency shifts (single atom index effect). The atom-field coupling during time $t$ produces an atomic-level dependent dephasing of the field and generates an entangled state given (for $\Omega / \delta \ll 1$ ) by Eq. (1) with $\phi=\Omega^{2} t / \delta$ [13].

The states $e$ and $g$ are circular Rydberg levels with principal quantum numbers 51 and 50 (transition frequency $\nu_{0}=51.099 \mathrm{GHz}$ ). They have a long radiative lifetime $(30 \mathrm{~ms})$ and a very strong coupling to radiation. The cavity $C$ is a Fabry-Pérot resonator with its axis normal to the atomic trajectory. It is made of two superconducting niobium mirrors (mirror distance $2.7 \mathrm{~cm}$; mode waist $6 \mathrm{~mm}$ ). $\Omega / 2 \pi$ is $24 \mathrm{kHz}$ [14]. The cavity $Q$ factor is $5.1 \times 10^{7}$ (photon lifetime $T_{r}=160 \mu \mathrm{s}$ ). The cavity is tuned by adjusting the mirror separation, thus varying $\delta / 2 \pi$ between 70 and $800 \mathrm{kHz}$. The effective interaction time $t$ is set to $19 \mu$ s by selecting atoms with a velocity of $400 \mathrm{~m} / \mathrm{s}$. For $\delta=100 \mathrm{kHz}, \phi$ is 0.69 radian which is an unusually large single atom index effect.

The setup is sketched in Fig. 2. It is cooled to $0.6 \mathrm{~K}$ by a $\mathrm{He}^{4}-\mathrm{He}^{3}$ cryostat making thermal radiation negligible (mean blackbody photon number in $C$ : 0.05). All $\mathrm{Rb}$ atoms effusing from the oven $\mathrm{O}$ are pumped out of the $F=3$ ground hyperfine level by a diode laser $L_{1}$ and optically repumped into this level by a diode laser beam $L_{1}^{\prime}$ oriented at $58^{\circ}$ relative to the atomic beam. With a proper tuning of $L_{1}^{\prime}$ in the Doppler profile, only atoms at $400 \pm 6 \mathrm{~m} / \mathrm{s}$ are prepared in $F=3$. The atoms are then excited into the circular state $e$ in box $B$ [15]. This pulsed process involves laser excitation from $F=3$ (lasers $L_{2}$ ) and prepares, on the average, 0.5 atom within a $2 \mu$ s time window, every $1.5 \mathrm{~ms}$.

Each circular atom is prepared in a superposition of $e$ and $g$ by a resonant microwave $\pi / 2$ pulse in a low $Q$ cavity $R_{1}$. It then crosses $C$ in which a small coherent field with an average photon number $n$ varying from

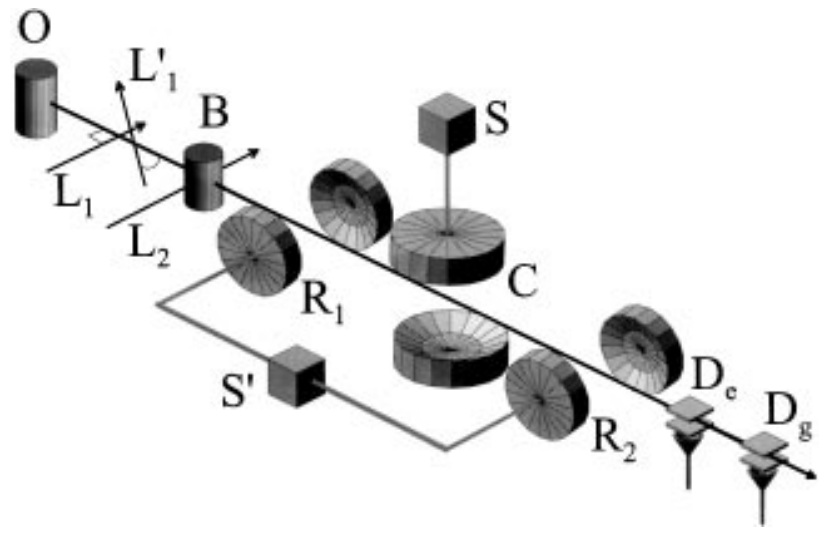

FIG. 2. Sketch of the experimental setup.
0 to 10 is injected by a pulsed source $S$ (see below how $n$ is measured). The field, which evolves freely while each atom crosses $C$, relaxes to vacuum before being regenerated for the next atom $\left(T_{r} \ll 1.5 \mathrm{~ms}\right)$. We make sure that the field is coherent by checking that $n$ is proportional to the square of the injection pulse duration. After leaving $C$, each atom undergoes another $\pi / 2$ pulse in a cavity $R_{2}$ identical to $R_{1} . R_{1}$ and $R_{2}$ are fed by a cw source $S^{\prime}$ whose frequency $\nu$ is swept across $\nu_{0}$. The atoms are finally counted in $e$ and $g$ by two field ionization detectors $\left(D_{e}, D_{g}\right.$; detection efficiency $40 \pm 15 \%$ ). With 50000 events recorded in $10 \mathrm{~min}$, the probability $P_{g}^{(1)}(\nu)$ to find the atom in $g$ as a function of $\nu$ is reconstructed.

Figure 3(a) shows the signal obtained when $C$ is empty $(\delta / 2 \pi=712 \mathrm{kHz}) . P_{g}^{(1)}(\nu)$ exhibits Ramsey fringes [16] typical of atoms subjected to two pulses separated by a time interval $T=230 \mu \mathrm{s}$. The fringes result from a quantum interference. The $e \rightarrow g$ transition can occur either in $R_{1}$ or in $R_{2}$ (atom crossing $C$ in $g$ or $e$ ). These two "paths" are indistinguishable, leading, in the final transition rate, to an interference term between the corresponding probability amplitudes. The phase difference between these amplitudes is $2 \pi\left(\nu-\nu_{0}\right) T$ so that $P_{g}^{(1)}(\nu)$ oscillates with the period $1 / T=4.2 \mathrm{kHz}$. The fringe contrast,

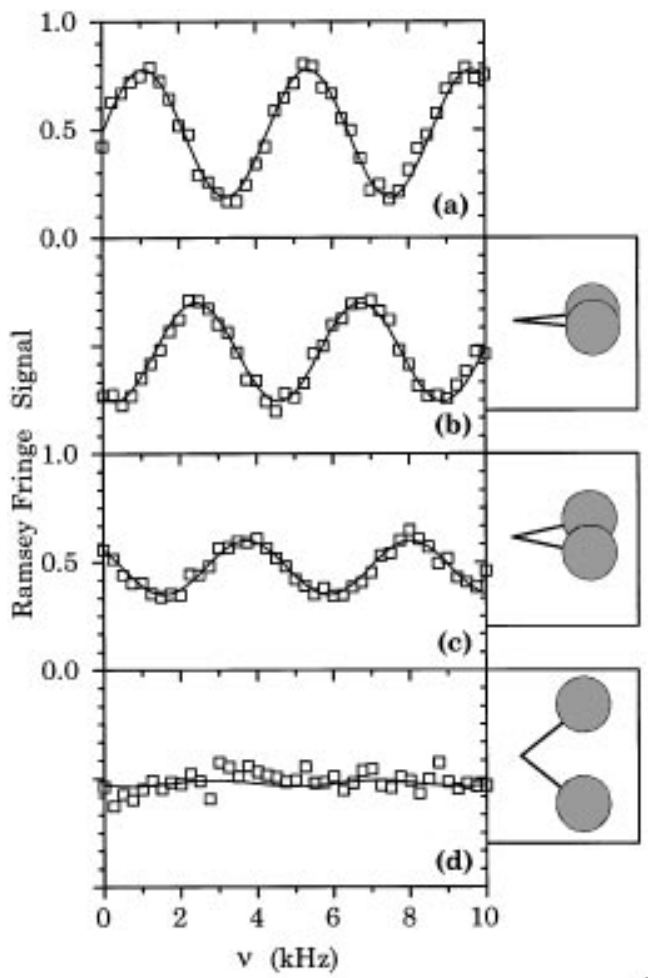

FIG. 3. $P_{g}^{(1)}(\nu)$ signal exhibiting Ramsey fringes: (a) $C$ empty, $\delta / 2 \pi=712 \mathrm{kHz}$; (b) $-(\mathrm{d}) C$ stores a coherent field with $|\alpha|=\sqrt{9.5}=3.1, \delta / 2 \pi=712,347$, and $104 \mathrm{kHz}$, respectively. Points are experimental and curves are sinusoidal fits. Insets show the phase space representation of the field components left in $C$. 
ideally $100 \%$, is reduced to $55 \pm 5 \%$ by various effects (static and microwave fields inhomogeneities between $R_{1}$ and $R_{2}$ over the $0.7 \mathrm{~mm}$ atomic beam diameter, finite atomic lifetime, atom count noise).

Figures 3(b) to 3(d) show the fringes for $\delta / 2 \pi=$ 712,347 , and $104 \mathrm{kHz}$ when there is a field in $C(n=$ 9.5; $|\alpha|=3.1)$. Two features are striking: when $\delta$ is reduced, the contrast of the fringes decreases and their phase is shifted. The fringe contrast and shift are plotted versus $\phi$ in Figs. 4(a) and 4(b) (points are experimental and lines are from theory; see below).

The fringe contrast reduction demonstrates the separation of the field state into two components and provides a measurement of $D^{2}$. When an atom leaves $C$, the system is prepared in the entangled state of Eq. (1), so that the field phase "points" towards $e$ and $g$ at the same time. The insets in Fig. 3 show the phase space representation of the two field components. When $\delta$ is large ( $\phi$ small), the measurement of the field phase would give no information on the state of the atom in $C$ (large overlap of the field components). The two "paths" (atom crosses $C$ in $e$ or $g$ ) are still indistinguishable and the contrast remains large [Fig. 3(b)]. When $\delta$ is reduced ( $\phi$ increased), the field contains more information about the atomic state in $C$. The two paths thus become partially distinguishable and the fringe contrast decreases [Fig. 3(c)]. It vanishes when the field components do not overlap at all [Fig. 3(d)].

It does not matter that the field is actually not measured. The mere fact that the atom leaves in $C$ an information which could be read out destroys the interference. We recognize the ingredients of a "which path" experiment illustrating the basic aspect of complementarity $[17,18]$. A simple calculation confirms the results of this discussion and shows that the fringe contrast is reduced by a factor equal to the modulus of the overlap integral $\left\langle\alpha e^{-i \phi} \mid \alpha e^{i \phi}\right\rangle=\exp \left(-D^{2} / 2\right) \exp (i n \sin 2 \phi)$. The corresponding line in Fig. 4(a) is in very good agreement with the measured points.

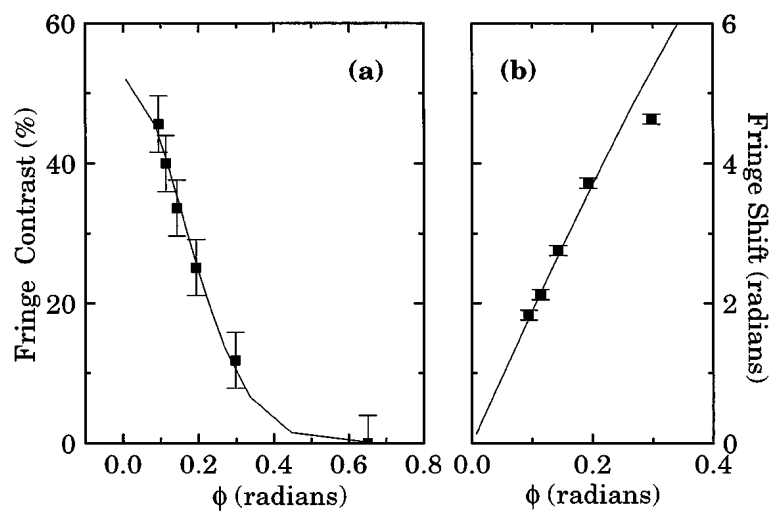

FIG. 4. Fringes contrast (a) and shift (b) versus $\phi$, for a coherent field with $|\alpha|=3.1$ (points: experiment; line: theory).
The same analysis shows that the phase of the fringes [Fig. 4(b)] is shifted by an angle equal to the phase of $\left\langle\alpha e^{-i \phi} \mid \alpha e^{i \phi}\right\rangle, n \sin 2 \phi$. The fringe phase shift is proportional to $n$ [19], which is determined from this set of data. The line on Fig. 4(b) corresponds to the best fitted value $n=9.5 \pm 0.2$.

The coherence between the two components of the state and its quantum decoherence were revealed by a subsequent two-atom correlation experiment, whose principle follows closely a proposal described in [20]. A first atom creates a superposition state involving two field components. A second "probe" atom crosses $C$ with the same velocity after a short delay $\tau$ and dephases again the field by an angle $\pm \phi$. The two field components turn into three, with phases $\pm 2 \phi$ and zero.

The "zero-phase" component is obtained via two different paths since the atoms may have crossed $C$ either in the $(e, g)$ or in the $(g, e)$ configurations. The probe atom "undoes" the phase splitting produced by the first one and recombines partially the state components. Since the atomic states are mixed after $C$ in $R_{2}$, the $(e, g)$ and $(g, e)$ paths are indistinguishable. As a result, there is an interference term in the joint probabilities $P_{e e}^{(2)}, P_{e g}^{(2)}, P_{g e}^{(2)}$, and $P_{g g}^{(2)}$ of detecting any of the four possible two-atom configurations. These probabilities can be calculated analytically, provided a few simplifying assumptions are made [21]. The difference between conditional probabilities $\eta=\left[P_{e e}^{(2)} /\left(P_{e e}^{(2)}+P_{e g}^{(2)}\right)\right]-\left[P_{g e}^{(2)} /\left(P_{g e}^{(2)}+P_{g g}^{(2)}\right)\right]$ is independent of $\nu$, except around $\phi=0$ and $\phi=\pi / 2$. Equal to 0.5 at short times $\tau$ when the quantum coherence is fully preserved, $\eta$ is shown to decay to 0 when the "first atom + field" system has evolved into a fully incoherent statistical mixture.

To measure $\eta$, the Rydberg state preparation pulse is replaced by a pair of pulses separated by $\tau$, varied from 30 to $250 \mu \mathrm{s}$. The sequence is, as before, repeated every $1.5 \mathrm{~ms}$ and statistics on double detection events are accumulated. Because of the low atom flux, the atom pair rate is 10 times smaller than the single atom count rate. For each delay $\tau, 15000$ coincidences are detected. Figure 5(a) shows $\eta$ versus $\nu$ for $n=3.3, \delta / 2 \pi=$ $70 \mathrm{kHz}$, and $\tau=40 \mu \mathrm{s}$. As predicted, a correlation signal with no statistically significant $\nu$ dependence is observed. A $\nu$-averaged $\eta$ value, $\bar{\eta}$, of $0.11 \pm 0.01$ is found for this $\tau$ value.

Figure 5(b) shows $\bar{\eta}$ versus $\tau$ (expressed in units of $\left.T_{r}\right)$ for $n=3.3$ and two different detunings $(\delta / 2 \pi=$ 170 and $70 \mathrm{kHz}$ ). The points are experimental and the lines theoretical. The theory includes higher order terms in $\Omega / \delta$ correcting the dephasings at $\delta=70 \mathrm{kHz}$, and incorporates the finite single atom fringe contrast (explaining the $\bar{\eta}$ value smaller than 0.5 at $\tau=0$ ).

The correlation signals decrease with time, revealing directly the dynamics of quantum decoherence. The agreement with the simple analytical model is excellent. 

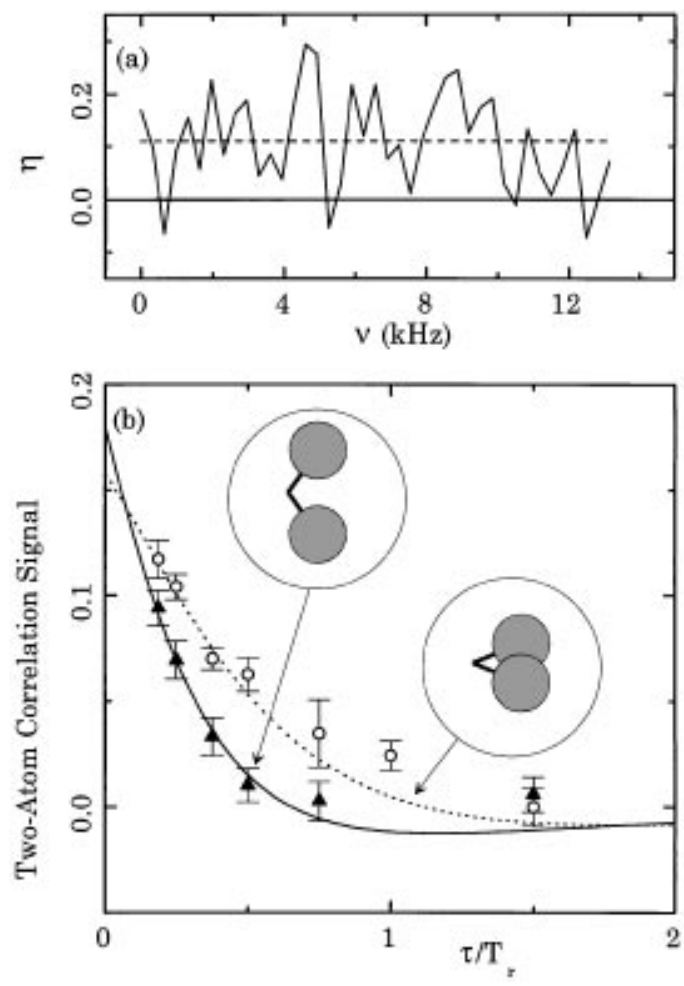

FIG. 5. (a) Two-atom correlation signal $\eta$ versus $\nu$ for $n=$ $3.3, \delta / 2 \pi=70 \mathrm{kHz}$, and $\tau=40 \mu \mathrm{s}$. (b) $\nu$-averaged $\eta$ values versus $\tau / T_{r}$ for $\delta / 2 \pi=170 \mathrm{kHz}$ (circles) and $\delta / 2 \pi=$ $70 \mathrm{kHz}$ (triangles). Dashed and solid lines are theoretical. Insets: pictorial representations of corresponding field components separated by $2 \phi$.

Most strikingly, we observe that decoherence proceeds at a faster rate when the distance between the two state components is increased. An effective decoherence time of $0.24 T_{r}$, much shorter than the photon decay time, is found for $\delta=70 \mathrm{kHz}$. A similar agreement with theory is obtained when comparing for the same $\delta / 2 \pi$ value $(70 \mathrm{kHz})$ the correlations signals corresponding to different $n$ values (5.1 and 3.3). We thus demonstrate the basic features of the decoherence theory on this simple model, namely, the fast evolution in a measurement process of the "atom + meter" state towards a statistical mixture and the increasing difficulty to maintain quantum coherence when the distance between the components of the mesoscopic superposition is increased. Using higher $Q$ cavities, we intend to increase $n$ further and to study decoherence processes occurring even faster on the scale of $T_{r}$. We can now continuously vary, from microscopic to macroscopic, the size of the meter in an ideal measurement process, allowing us to explore the elusive boundary between the quantum and classical worlds.
We thank P. Goy for assistance with microwave technology and $\mathrm{AB}$ Millimetre for the loan of equipment. This work was supported in part by EEC (Grant No. ERBCHRXCT930114).

*Laboratoire de l'Université Pierre et Marie Curie et de l'ENS, associé au CNRS (URA18).

[1] J. A. Wheeler and W.H. Zurek, Quantum Theory of Measurement (Princeton Univ. Press, Princeton, NJ, 1983).

[2] E. Schrödinger, Naturwissenschaften 23, 807, 823, 844 (1935); reprinted in English in [1].

[3] J. von Neumann, in Matematische Grundlagen der Quantenmechanik (Springer, Berlin, 1932); reprinted in English in [1].

[4] W. H. Zurek, Phys. Today 44, No. 10, 36 (1991).

[5] W. H. Zurek, Phys. Rev. D 24, 1516 (1981); 26, 1862 (1982); A. O. Caldeira and A. J. Leggett, Physica (Amsterdam) 121A, 587 (1983); E. Joos and H. D. Zeh, Z. Phys. B 59, 223 (1985); R. Omnès, The Interpretation of Quantum Mechanics (Princeton University Press, Princeton, NJ, 1994).

[6] R. J. Glauber, Phys. Rev. 131, 2766 (1963).

[7] D. F. Waals and G.J. Milburn, Phys. Rev. A 31, 2403 (1985).

[8] M. Brune et al., Phys. Rev. A 45, 5193 (1992).

[9] C. Monroe et al., Science 272, 1131 (1996).

[10] B. Yurke and D. Stoler, Phys. Rev. Lett. 57, 13 (1986); B. Yurke et al., Phys. Rev. A 42, 1703 (1990); G. Milburn, ibid. 33, 674 (1986); V. Buzek et al., Phys. Rev. A 45, 8190 (1992).

[11] C. N. Savage et al., Opt. Lett. 15, 628 (1990).

[12] R. G. Hulet and D. Kleppner, Phys. Rev. Lett. 51, 1430 (1983).

[13] S. Haroche and J.M. Raimond, in Cavity Quantum Electrodynamics, edited by P. Berman (Academic Press, New York, 1994), p. 123.

[14] M. Brune et al., Phys. Rev. Lett. 76, 1800 (1996).

[15] P. Nussenzveig et al., Phys. Rev. A 48, 3991 (1993).

[16] N.F. Ramsey, Molecular Beams (Oxford Univ. Press, New York, 1985).

[17] M. O. Scully et al., Nature (London) 351, 111 (1991); S. Haroche et al., Appl. Phys. B 54, 355 (1992).

[18] T. Pfau et al., Phys. Rev. Lett. 73, 1223 (1994); M. S. Chapman et al., Phys. Rev. Lett. 75, 3783 (1995).

[19] M. Brune et al., Phys. Rev. Lett. 72, 3339 (1994).

[20] L. Davidovich et al., Phys. Rev. A 53, 1295 (1996).

[21] The calculation - to be published - generalizes to arbitrary $\phi$ values the derivation of [20], which was restricted to $\phi=\pi / 2$. It describes independently atom-field interaction and relaxation and neglects fluctuations of atomic velocity as well as of atom number (possibility of two atoms in each preparation pulse). 